\title{
CHORIOCARCINOMA ASSOCIATED WITH ECTOPIC PREGNANCY. A CASE REPORT
}

\author{
Manandhar $T^{1^{*}}$, Upreti $R^{2}$, Sitaula $S^{1}$, Thapa $B D^{1}$, Thakur $A^{3}$
}

\section{Affiliation}

1. Assistant Professor, Department of Obstetrics and Gynaecology, B.P Koirala Institute Of Health Sciences, Dharan, Nepal

2. Resident, Department of Obstetrics and Gynaecology, B.P Koirala Institute Of Health Sciences, Dharan, Nepal

3. Additional Professor, Department of Obstetrics and Gynaecology B.P Koirala Institute Of Health Sciences, Dharan, Nepal

\section{ARTICLE INFO}

\section{Received : 02 May, 2019 \\ Accepted : 14 August, 2019}

Published : 31 August, 2019

(C) Authors retain copyright and grant the journal right of first publication with the work simultaneously licensed under Creative Commons Attribution License CC - BY 4.0 that allows others to share the work with an acknowledgment of the work's authorship and initial publication in this journal.

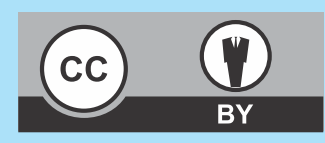

CR 29

DOI: http://dx.doi.org/10.3126/bjhs.v4i3.27045

* Corresponding Author

Dr Tara Manandhar

Assistant Professor

Department of Obstetrics and Gynaecology

B.P Koirala Institute of Health Sciences, Dharan, Nepal

Email: tara2073m@gmail.com

ORCID ID: https://orcid.org/0000-0003-2607-510X

\section{ABSTRACT}

Choriocarcinoma is an uncommon malignant neoplasia and even rarer is the Choriocarcinoma associated with ectopic pregnancy. Here, we present a case of Choriocarcinoma in a young lady who had undergone laparoscopic salphingectomy for rupture ectopic pregnancy, and presenting after 2 month in hemorrhagic shock requiring urgent laparotomy.

\section{KEY WORDS}

Choriocarcinoma, ectopic pregnancy, gestational trophoblastic neoplasia.

\section{Citation}

Manandhar T, Upreti R, Sitaula S, Thapa BD, Thakur A. Choriocarcinoma Associated with Ectopic Pregnancy. A Case Report. BJHS 2019;4 (3)10:873-876 


\section{INTRODUCTION}

Choriocarcinoma, a persistent gestational trophoblastic neoplasia (GTN), is a highly malignant tumor arising from the chorionic epithelium. About 3 to $5 \%$ of Choriocarcinoma occurs after molar pregnancy, where there is malignant transformation of chorionic tissue. ${ }^{1}$ GTN after the molar pregnancy can either be Invasive mole or Choriocarcinoma, but following non-molar pregnancy, GTN is always a Choriocarcinoma. Though themolar pregnancy is the commonest cause for Choriocarcinoma, it can sometimes occur following term pregnancy, spontaneous abortion, and even after ectopic pregnancy. ${ }^{1,2}$ Choriocarcinoma arising inectopic pregnancy is an extremely rare event with the incidence of 1 in 5333 tubal pregnancies. ${ }^{1,3}$ Choriocarcinoma usually present with nonspecific symptoms and can mimic with different other clinical presentations like Hemorrhagic ovarian cyst, Tubo-ovarian abscess, Ovarian torsion, and ectopic pregnancy. Approximately 30\% cases present with advance disease, having metastasis to various organs like lungs, vagina, liver and brain. Therefore, high index of suspicion is required for diagnosis. Delay in diagnosis and treatment can increase the morbidity and mortality. Different investigations like USG and Serial measurement of $\beta$ HCG level can help in early diagnosis and prompt initiation of chemotherapy for the better prognosis. Different treatment modalities including surgery and/or chemotherapy depends upon the age, parity and severity of disease. ${ }^{3}$ Here we present a case who had undergone a laparoscopic salpingectomy for tubal ectopic pregnancy and was later diagnosed to have the Choriocarcinoma.

\section{CASE DESCRIPTION}

A 19 years, Para 0, Abortion 1, Ectopic 1, Live 0 presented with Pain lower abdomen for 2 days, Unable to pass urine and per vaginal bleeding for 1 day. Two months back she had undergone Laparoscopic right sided salpingectomy for Ruptured Ectopic pregnancy. But unfortunately, the specimen was not sent for Histopathological examination due to some family issues. On examination she was pale, febrile, and had tachycardia. Foley catheter was in situ draining frank blood. Per abdominal examination revealed a tender boggy mass in the suprapubic region. On per speculum examination, the cervix was normal but there was profuse whitish discharge. There was tenderness over bilateral fornices on bimanual examination. Urinary for pregnancy test was positive. Laboratory investigations were within normal limit except for low hemoglobin level. Transabdominal ultrasonography showed a large solid cystic mass in the right adnexa. Patient was planned for Emergency Exploratory laparotomy. Intraoperatively, there was a fragile, hemorrhagic and necrotic growth with erosions involving the right sided interstitium of fallopian tube, right cornua of the uterus and was invading the posterior wall of the urinary bladder (figure 1 and 2). Hence, Hysterectomy along with Cystostomy was done (figure 3). Inside bladder there was a Jet like projection of blood vessel in the posterior wall of the bladder wall along with blood clot. The Blood clot was evacuated and DJ stenting along with suprapubic catheterization was done. Histopathological examination reportrevealed Choriocarcinoma (figure 4). Patient falls under low risk on WHO prognostic scoring, so she was started with single cycle chemotherapy with Methotrexate and Folinic acid. Patient hadcompleted her $5^{\text {th }}$ cycle of single agent Chemotherapyand is under regular follow up with serial $\beta$ HCG level.

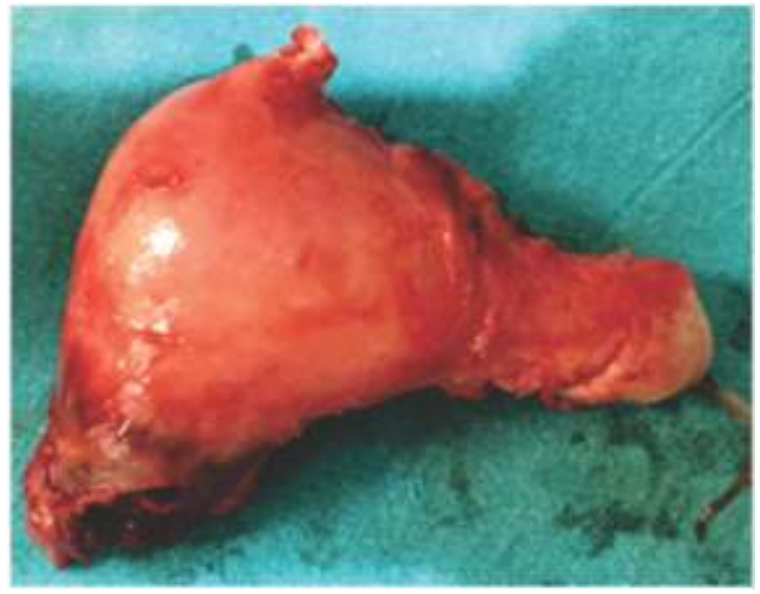

Figure 1: The uterus with necrotic and hemorrhagic growth in the rt cornua

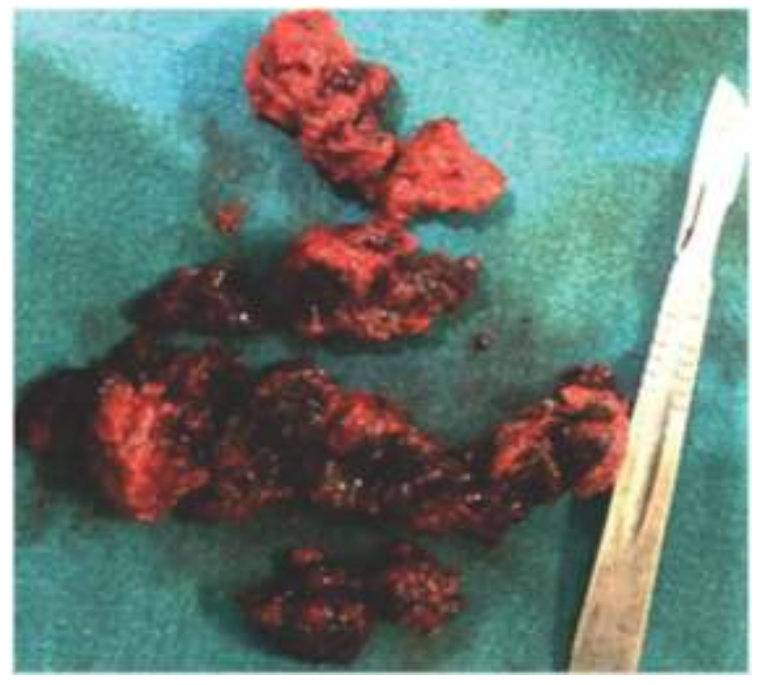

Figure 2: Excised necrotic growth

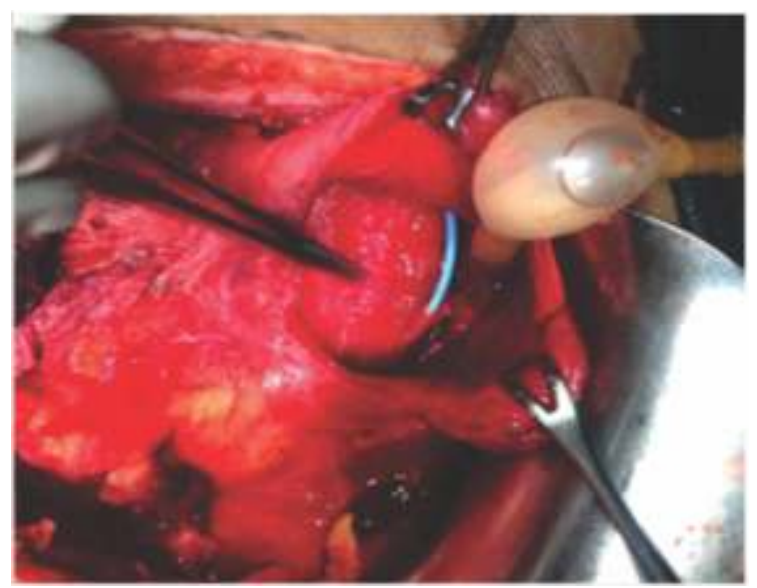

Figure 3: Intraoperative cystotosmy with DJ sent in situ 


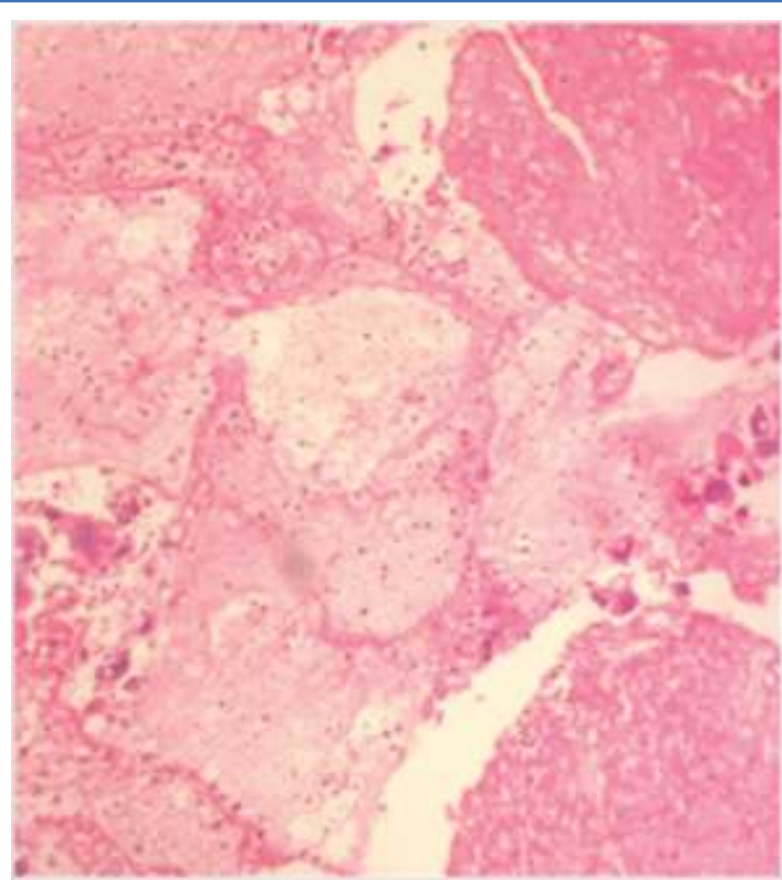

Figure 4: Rt cornua of uterus shows masses and sheets of trophoblastic cells with masked atypia in the form of nuclear enlargement, hyperchromasia and prominent nucleoli, invading the surrounding muscle. The cytotrophoblasts and intermediate trophoblasts are in sheets seperated by syncytiotrophoblast forming dismorphic growth pattern.

\section{DISCUSSION}

Choriocarcinoma is a persistent gestational trophoblastic neoplasia, and is a highly malignant tumor arising from the chorionic epithelium. The most common site of origin of Choriocarcinoma is uterus and occurs mainly after the malignant transformation of molar pregnancy, but sometimes it has been seen after term pregnancy, spontaneous abortion or ectopic pregnancy at various sites. ${ }^{1,2,4}$ Choriocarcinoma after an ectopic pregnancy $(0.76 \%$ to $4 \%)$ is a rare entity. ${ }^{3,6}$ The reported incidence of ectopic tubal choriocarcinoma is approximately one in 5333 tubal pregnancies and 1.5/1,000,000 births. ${ }^{1,3-5,7}$ The occurrence of choriocarcinoma in very variable ranging 5 weeks to 15 years after gestation and can occur even after menopause. ${ }^{1,3}$ The incidence of choriocarcinoma after ectopic gestation is less but is very aggressive disease with around $30 \%$ presenting with metastasis at time of diagnosis. Lung is the most common site of metastasis (80\%) followed by vagina (30\%), liver and brain $(10 \%) .{ }^{1,5}$ Choriocarcinoma, including those arising in an ectopic location, is highly responsive to chemotherapy, even in advanced stages. Early identification of the disease is very important as it carries excellent prognosis, however, it is very challenging as most of them present with various nonspecific symptoms. Numerous case reports have been published in various journals regarding the unusual clinical presentations of this cancer: amenorrhea, vaginal bleeding, pelvic pain with increase in serum $\beta$ HCG level and can mimic ectopic pregnancy. Also, the presentations may be confused with other clinical conditions like Hemorrhagic ovarian cyst, Tubo-ovarian abscess, Ovarian torsion. ${ }^{2,3}$ There are many techniques both invasive and noninvasive which help to minimize this diagnostic dilemma and aid in the diagnosis of ectopic gestational choriocarcinoma. Ultrasonography both Transabdominal and Transvaginal, Color flow Doppler, MRI, and even hysteroscopy plays a significant role in the diagnosis of gestational choriocarcinoma. ${ }^{3-5}$ Overall, the gold standard for diagnosing choriocarcinoma is the histopathological evaluation. There will be columns of trophoblastic cells, commonly separated by coagulated blood that invades muscular tissue in case of choriocarcinoma. Combination of P57KP2 immunostaining and DNA polymorphism analysis has been significant in determining the origin of gestational and non-gestational tubal choriocarcinoma. ${ }^{4}$ Management includes local resection of tumor combined with chemotherapy or complete surgical resection with adjuvant chemotherapy. But along with that one should be monitored with serial measurement of $\beta$ - HCG. These tumors are chemo-sensitive and regimen depends on the risk score of the patient. The Prognostic scoring system classify gestational trophoblastic neoplasia into low risk ( risk score $=<6$ ) and high risk (risk score >6) depending upon various parameters: Age, antecedent pregnancy, time interval between antecedent pregnancy and the start of chemotherapy, pretreatment $\beta$ - HCG level, tumor size, site of metastasis, number of metastasis, and prior failed chemotherapy. ${ }^{6}$ Lowrisk GTN patients are treated with single agent methotrexate or actinomycin D protocols. Multi agent chemotherapy is required only in the cases where there is a significant elevation in hCG level, development of metastasis or resistance to sequential single agent chemotherapy. Higher risk score (5-6) and clinicopathological diagnosis of choriocarcinoma are both associated with an increased risk of resistance to single agent chemotherapy and there should me lower threshold for multi agent chemotherapy in this subset of low risk patients. High risk GTN are treated with the multi agent chemotherapy (EMA-CO chemotherapy, but there are subset in the group also called as ultra-high risk GTN (risk score 13 or more, liver/brain or extensive metastasis) do poorly when treated with the first line chemotherapy. Besides chemotherapy, surgery has important role in controlling the bleeding and in resection of the isolated drugresistant tumor. ${ }^{4,6}$

\section{CONCLUSION}

Choriocarcinoma is a highly malignant tumor but is rare, and even rarer is presenting after ectopic pregnancy. It usually present with bleeding, if not hemorrhagic shock, and may mimic the other common conditions. It should be suspected in patient with high level of beta HCG. Early surgical intervention followed by chemotherapy depending on the risk-score, has favorable outcome.

\section{CONFLICT OF INTEREST}

None 


\section{REFERENCES}

1. Mangla M, Singla D, Kaur H, Sharma S. Unusual clinical presentations of choriocarcinoma: A systematic review of case reports. Taiwan J Obstet Gynecol. 2017;56(1):1-8.

2. Karaman E, Çetin O, Kolusarı A, Bayram I. Primary tubal choriocarcinoma presented as ruptured ectopic pregnancy. J Clin Diagnostic Res. 2015;9(9):QD17-8.

3. Meddeb S, Rhim MS, Zarrouk W, Bibi M, Yacoubi MT, Khairi H. Unusual gestational choriocarcinoma arising in an interstitial pregnancy. Int J Surg Case Rep. 2014;5(11):787-8.

4. Mehrotra S, Singh U, Goel M, Chauhan S. Ectopic tubal choriocarcinoma: a rarity. BMJ Case Rep. 2012 Nov 11;2012(nov09 1):bcr-2012-006318-bcr-2012-006318.

5. Han V, Kaye S. A Rare Case of Gestational Choriocarcinoma Presenting as Cornual Ectopic Pregnancy. J Obstet Gynaecol Canada. 2018;40(3):351-3.

6. Ngan HYS, Seckl MJ, Berkowitz RS, Xiang Y, Golfier F, Sekharan PK, et al. Update on the diagnosis and management of gestational trophoblastic disease. Int J Gynecol Obstet. 2018;143:79-85.

7. Petre I, Bernad E, Mureşan A, Bordianu A, Bernad SI, Băcean O, et al. Choriocarcinoma developed in a tubal pregnancy - A case report. Rom J Morphol Embryol. 2015;56(2):871-4. 